\title{
Bronchial carcinoid tumor: case report
}

\begin{abstract}
Bronchial carcinoid tumors are an uncommon group of lung tumors. The first report of BCT was by Laennac in 1831 . They represent about $1 \%$ of all primary lung tumors and about $80 \%$ are centrally located, within the lobar and segmental bronchi. ${ }^{1,2}$ BCTs were initially classified as benign tumors but now more appropriately classified as malignant tumors. They arise from the kulchitsky cells in the bronchial mucosa, more often in the cartilaginous portion of the tracheobronchial tree, covered by intact epithelium. They are highly vascular and may have a broad based or polypoid attachment. BCTs are capable of secreting biologically active substances. Over $80 \%$ of the tumors express somatostatin receptor subtype 2 . The cause of BCTs is not proven and there is no strong association with smoking and environmental carcinogens. Familial BCTs have been reported with or without multiple endocrine neoplasia type 1. Leothela et al., ${ }^{3}$ attributed BCTs to loss of heterogeneity in multiple chromosomes.

The World Health Organization classifies BCTs into 2 groups. Typical BCTs are well differentiated with very low malignant potential, have less than 2 mitotic figures per 10high power field and no evidence of necrosis. Atypical BCT have more than 2-10 mitotic figures per 10 high power field and evidence of necrosis. Typical BCTs are about four times more common than atypical BCTs. We are reporting a case of typical bronchial carcinoid tumor since none has been reported before in the sub-region, to the best of our knowledge.
\end{abstract}

Keywords: bronchial carcinoid tumor, diagnosis, treatment, outcome, West Africa
Volume I Issue I - 2014

\section{Obinna Orakwe}

Federal Medical Centre, University of Nigeria Teaching Hospital, Nigeria

Correspondence: Obinna Orakwe, Federal Medical Centre, University of Nigeria Teaching Hospital, Asaba, Nigeria, Tel +2348037727116,Email obinnaorakwe@ymail.com

Received: July 21, 2014 | Published: July 28, 2014

\section{Case report}

A 25year old male from SE, Nigeria presented at the cardiothoracic unit with a 4years history of cough, hemoptysis and shortness of breath. He did not lose weight or sweat excessively. He has never smoked and is not a known asthmatic. He had a complete course of anti-tuberculosis drugs but there was no improvement. He looked well nourished and active. There was a dull percussion note and a reduced air entry in the left upper lung zone. Chest x-ray showed a significant left hilar opacity (Figure 1). Chest CT scan revealed an oval mass in the left upper lobe with a widest diameter of $6.19 \mathrm{~cm}$ obstructing the left upper lobar bronchus with collapse of the upper lobe segments. There was no enlarged left hilar or mediastinal lymph nodes (Figures 2-4). A trans-thoracic needle biopsy reported typical BCT (Figure 5). He had a left upper lobectomy with regional lymph node dissection through a left postero-lateral thoracotomy. The left lower lobe was free of any tumor (Figure 6). Left upper lobar mass and hilar lymph nodes were sampled and reported as typical BCT (Central type) with tumor free resection margins and no lymph node involvement (Figure 7). They tumor cells strongly expressed broad spectrum cytokeratins, synaptophysin, CD 56 and NSE (Figures 8-11).

The patient presented with the classical features widely described in the literature. There is no such report from our sub-region so far. The reported global incidence is about $0.2-3$ per 100,000 per year and appears to be rising because of improved imaging techniques. ${ }^{2}$ There is no wide sex or racial variation. Typical BCTs have been reported in all age groups but peak around $4^{\text {th }}$ decade while atypical BCTs occur more around the $5^{\text {th }}$ decade..$^{1,2}$ Central tumors are usually symptomatic with features of bronchial obstruction, irritation and ball valve effect for polypoid tumors, while peripheral tumors are usually asymptomatic. They can present with Carcinoid syndrome, Cushing's syndrome, acromegaly, etc. Chest x-ray reveals the tumor mass, parenchyma changes due to obstruction and/or evidence of pleural effusion. Chest CT scan better defines the tumor mass, location, relationship with the tracheobronchial tree and the lymph node status. Tumor portions outside the airway are better appreciated than with bronchoscopy and there is a greater contrast enhancement for BCTs than with other lung tumors due to higher vascularity. Bronchoscopy offers the best choice for tissue diagnosis. Tumor resection, laser or photodynamic therapy can be offered at bronchoscope. Trans-thoracic needle biopsy VATS or thoracotomy are other options for tissue diagnosis. Histology is by routine light microscopy and confirmed by Immunohistochemistry. Surgery is the mainstay of treatment. ${ }^{2,4}$ Lung resection and lung parenchymal sparing bronchoplastic procedures with regional lymph node dissection have been offered with excellent outcomes especially for typical BCTs. Chemotherapy and radiotherapy are not yet convincing. ${ }^{2} 5$ years survival for typical BCTs is over $80 \%$ and about $50 \%$ for atypical BCTs. Recurrence is very low, about $3-5 \%$, and is usually due to incomplete resection. ${ }^{1,4}$

BCTs commonly affect the same age group affected by other more common chronic lung conditions such as pulmonary tuberculosis. Bora et al., ${ }^{5}$ in 2012 reported BCT as a rare differential of pulmonary tuberculosis. Dixit et al., ${ }^{6}$ in 2009 reported a case of BCT with concomitant pulmonary tuberculosis. This underscores the need for a good index of suspicion with the resurgence of pulmonary tuberculosis and the rising incidence of BCTs. Diagnosis is missed or delayed even in symptomatic patients due to a low index of suspicion, paucity of knowledge and lack of facilities for accurate diagnosis. Faduyile et al., in 2012 reported a post-mortem case of a 53year old man who died from atypical bronchial characinoid tumor while being wrongly treated for pulmonary tuberculosis. In many reports diagnosis is made more than 1year after presentation. This may be worse in our setting due to earlier highlighted reasons, poor referral system and the high tendency to resort to alternative health care services. 


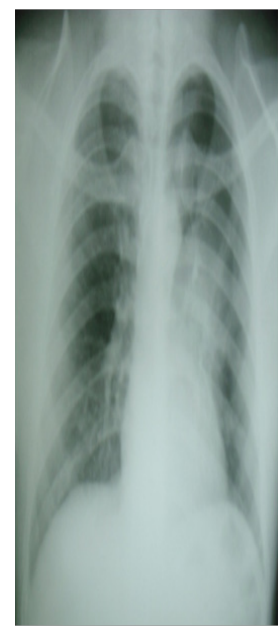

Figure I CXR showing left hilar opacity.

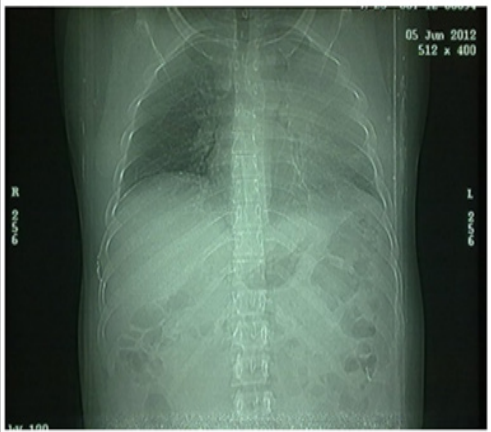

Figure 2 Chest CT scanogram showing opacification of the left hemi-thorax.

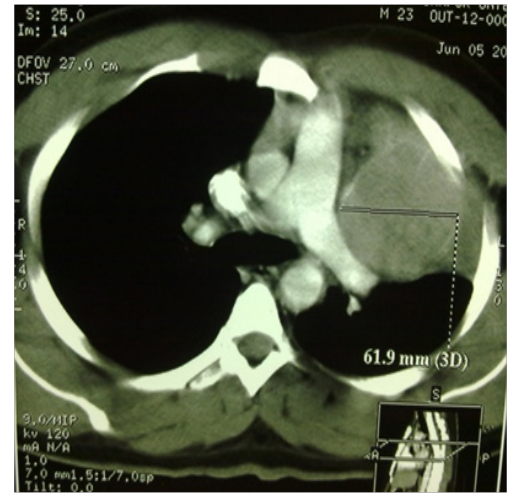

Figure 3 Axial CT at the level of tracheal bifurcation showing a left upper lobe mass.

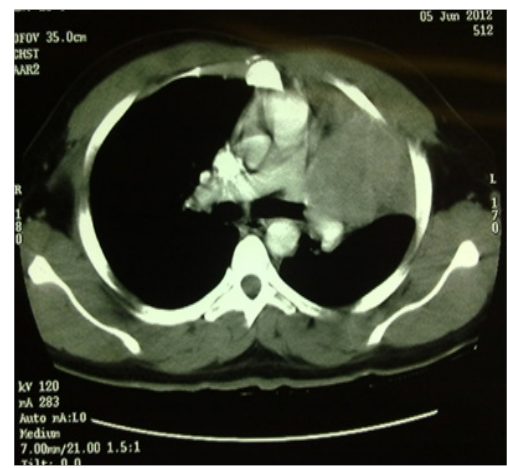

Figure 4 Axial CT showing a close relationship with the left main stem bronchus.

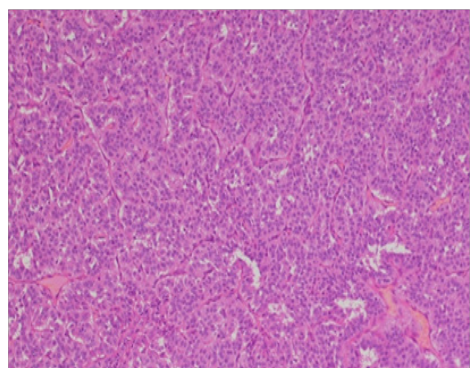

Figure 5 Photomicrograph showing proliferation of uniformly small round to oval cells forming nests, rosettes and small acinar structures. The tumor stroma is scanty \& vascular. There are no foci of tumor necrosis $(H$ \& E Stain $x(0)$.

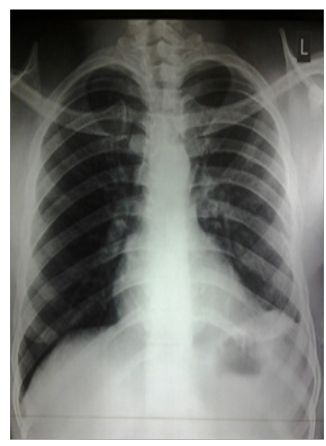

Figure 6 Post-op chest x-ray.The left lower lobe is not affected by the tumor and fills up the left hemi-thorax.

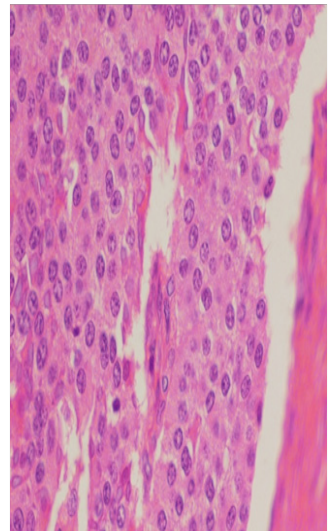

Figure 7 Photomicrograph showing proliferation of uniformly small round to oval cells forming nests, rosettes and small acinar structures. The tumor stroma is scanty \& vascular. There are no foci of tumor necrosis ( $H$ \& $E$ Stain $\times 40)$.

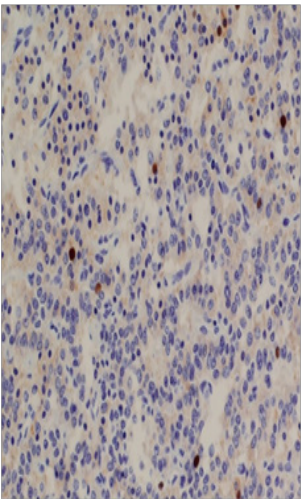

Figure 8 Photomicrograph showing poor immunohistochemical staining of the carcinoid tumor cells for $\mathrm{Ki}-67$ (x 400). The $\mathrm{Ki}-67$ index is about I\%. 


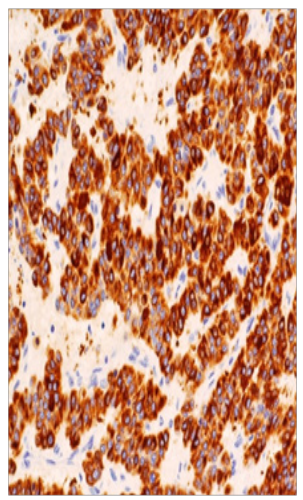

Figure 10 Photomicrograph showing strong immunohistochemical staining of the carcinoid tumor cells for synaptophysin (x 200).

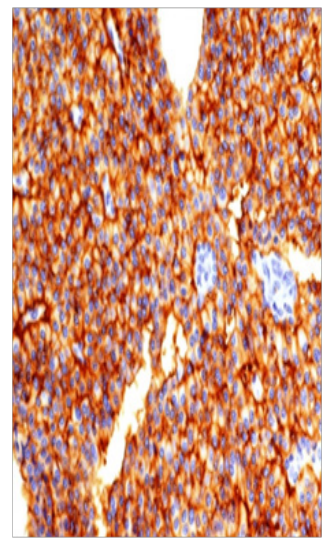

Figure I I Photomicrograph showing strong immunohistochemical staining of the carcinoid tumor cells for CD56 (N-CAM) x 200).

\section{Conclusion}

BCTs also occur in our sub-region. A good index of suspicion and knowledge of the tumor is very important for a good outcome.

\section{Acknowledgements}

None.

\section{Conflict of interest}

The author declares no conflict of interest.

\section{References}

1. Detterbeck FC. Management of carcinoid tumors. Ann Thorac Surg. 2010;89(3):998-1005.

2. Mancini MC, Milliken JC. Carcinoid lung tumors. 2013.

3. Leothela PD, Jauch A, Holtgreve-Grez H, et al. Genetics of neuroendocrine and carcinoid tumors. Endocr Relat Cancer. 2003;10(4): 437-500.

4. Rea F, Rizzardi G, Zuin A, et al. Outcome \& surgical strategy in bronchial carcinoid tumors: single institution experience with 252 patients. Eur J Cardiothoracic Surg. 2007;31(2):186-191.

5. Bora MK, Vithiavathi S. Primary bronchial carciniod: A rare differential diagnosis of pulmonary koch in young adult patient. Lung India. 2012;29(1):59-62.

6. Dixit R, Gupta R, Yadav A, et al. A case of pulmonary carcinoid tumor with concomitant tuberculosis. Lung India. 2009;26(4):133-135.

7. Faduyile FA, Sanni DA, Soyemi SS, et al. Atypical carcinoid tumor of the lung: An enigma. World Journal of Pathology. 2012;3(1):9. 